\title{
Electronic excitation of atoms by positron impact using the scaling born approach
}

\author{
Jorge L. S. Lino \\ Assessoria e Orientação Estudantil - AOE, \\ Cep: 12222-000, no 2375, São José dos Campos, São Paulo, Brasil. \\ Received 31 May 2018; accepted 11 July 2018
}

\begin{abstract}
We consider the efficiency of the scaling Born positron (SBP) approach, in calculating reliable integral cross sections (ICS) for positron impact excitation of electronic states in atoms. We will demonstrate, using specific examples as $\mathrm{H}, \mathrm{He}, \mathrm{Hg}$, and $\mathrm{Mg}$, that this relatively simple procedure can generate quite accurate ICS when compared with more sophisticated methods. In the absence of the experimental data, comparisons are made with analogous electron scattering.
\end{abstract}

Keywords: Born; positron; scaling.

PACS: $34.80 . D p$

\section{Introduction}

Similar to the electron, its anti-particle (positron) also has important scientific and technological applications in a large variety of fields. A thorough presentation of the many applications of positrons can be found, for instance, in the book by Charlton and Humberston [1]. This includes astrophysics, solar physics, bio-medicine (both diagnostics and therapy) and materials science (defect studies and crystallography). From a more fundamental perspective, positrons are essential in the formation of antihydrogen, understanding elementary particle and positronium (Ps) physics, as well as in the investigation of positron binding to ordinary matter, i.e. neutral atoms [1]. Resonances in electron-impact on atoms and molecules are well-known; however, the situation with respect to positrons is not as clear [1]. Positron binding energies have been measured for a large variety of small targets [1], although only a few calculations are available[1]. Positron scattering in the gases phase constitutes a sensitive test for atomic interactions. The static potential between the incoming electron and the fixed charge distribution in an atom is attractive. Positrons inside an atom experience a repulsive static interaction from the positive nucleus only partially screened by electrons. The opposite sign of the static interaction for positrons cause a kind of compensation between the two parts of the potential (static + polarization) and small adjustments of these parts cause big differences in cross sections. New scattering measurements are very important for comparison, setting new standards for both theoreticians and experimentalists [1,2]. Indeed one rationale for the present investigation is to try and shed more light on this state of affairs. In the last few years, there have been several theoretical activities concerning the positron-atom scattering at several energies [3]. Most of the work produced was based on ab initio methods [3] and also classical collision theory [3]. However, each of these models works only on a limited range of targets and these calculations are very time consuming, limiting the domain of applicability of such models.

In the present work we present a study on the simple scaling of plane wave Born cross section which was created for positron-impact excitations of targets in general [4]. The study is based on the traditional first Born approximation (FBA). The FBA still is used as the starting point in several studies, because (a) the plane wave is the correct wave function at infinity for an positron colliding with a target, and (b) it is the simplest collision theory that uses target wave function explicitly. Validating a scaling method for FBA cross sections of atoms requires two initial ingredients: (i) the Born integral cross sections themselves; (ii) reliable experimental or theoretical optical oscillator strengths. The called BE $f$ scaling approach was found by Kim [5] to convert the FBA to reliable cross sections comparable to accurate excitation cross sections at all incident electron energies above threshold. The BE $f$-scaling described by Kim [5] correct the deficiencies of FBA into simple functional forms that depend on a few atomic properties. Cross sections for positron and electron impact are virtually identical at high energies and if the basic dynamical ingredients for this evidence is the FBA, then it is possible extend the analysis developed by Kim [5] to more complicated systems, as positron-atom scattering (this is a important consideration and can be significant for studies using positron as incident particle) [4]. One of the complications created by the use of positron as incident particle is the existence of additional positronium channels which are not present in the case of electron scattering. Thus, we will present a study of the SBP approach without Ps channel. The goal of the present scaling method is to provide a simple theoretical method to calculate excitation cross sections comparable not only to reliable experimental data, but also to more sophisticated theories. To our knowledge, this study represents the first attempt to establish a theoretical formulation for positron scattering using the called scaling Born positron (SBP), i.e., a version for positron of originally $\operatorname{BE} f$-scaling proposed by Kim [5] for electron scattering.

In Sec. 2 we identify changes necessary to transform the model proposed by Kim [5] (electron scattering) and the present model for positron scattering. In Sec. 3 we discuss the application of the method for excitation of $\mathrm{H}, \mathrm{He}, \mathrm{Hg}$, and Mg. Conclusions are presented in Sec. 4. 


\section{Theory}

The scaling Born approximation described by Kim [5] for excitation of neutral atoms is applicable to dipole-allowed excitations, and use atomic properties as excitation energy, ionization energy, and the dipole $f$ value that can be obtained, in principle, from accurate wave functions. Since scaled cross sections are based on the plane wave Born approximation (FBA), they do not account for the resonances often found near the excitation thresholds. We will see that our method for positron scattering not only reduces the cross sections magnitude at low energy, but also shifts the peak to a high energy than the peak of the unscaled FBA, while keeping the high energy validity of the Born approximation intact. In a generic form, first order Born cross sections $\sigma_{\text {Born }}(E)$ for inelastic collisions are written as [4,5]

$$
\sigma_{\text {Born }}\left(E_{0}\right)=4\left(\frac{\pi}{E}\right) R a_{0}^{2} F_{\text {Born }} E_{0}
$$

where $a_{o}$ is the Bohr radius, $R$ is the Rydberg energy, $E_{o}$ is the incident positron energy, and $F_{\mathrm{Born}} E_{0}$ is the collision strength (multiplied by a constant to be consistent with the standard definition of the collision strength). The SBP cross sections are described as

$$
\begin{aligned}
\sigma_{\mathrm{SBP}} & =\left\{\left(f_{\text {accur }} / f_{\text {Born }}\right)\right. \\
& \left.\times\left(E_{0} /\left[\left(E_{0}+E_{\mathrm{ps}}+E_{\mathrm{exc}}\right)\right]\right)\right\} \cdot \sigma_{\mathrm{Born}}
\end{aligned}
$$

where $E_{0}$ is the incident positron energy, $f_{\text {accur }}$ is an accurate dipole value from experiments or from accurate wavefunctions, and $f_{\text {Born }}$ is the dipole value from the same wavefunctions used to calculate the unscaled Born cross section $\left(\sigma_{\text {Born }}\right), E_{\mathrm{ps}}$ is positronium energy, and $E_{\mathrm{exc}}$ excitation energy. The amplitude for the FBA is given by

$$
f_{\text {Born }}=-(1 / 2 \pi) \int d^{3} r e^{i(k i-k f)} \cdot r\left\langle\Phi_{f}|V| \Phi_{i}\right\rangle
$$

In Eq. (3), $V$ is the positron-target interaction potential while $\Phi_{i}$ and $\Phi_{f}$ are initial and final electronic states of the target, respectively. Scattering amplitude obtained from FBA are valid for high-energy static calculations, i.e., situations where the target wave functions can be considered frozen. For inelastic scattering, the FBA can be justified only by a favorable comparison with more elaborate methods. As we will see, the SBP approach represent a considerable improvement on the FBA, indicant cross sections with the same order of magnitude as the more sophisticated calculations. The $\left(E_{\mathrm{ps}}+E_{\mathrm{exc}}\right)$ term used in Eq. (2) is a constant for each $Z$ of the target and is related with the energy change of the incident positron in the field of the nucleus and the bound electrons of the target. From low-energy investigations, it is expected that a slow positron can distort considerably the electronic cloud. A connection between this consideration and the Eq. (2) may be related to the polarizability of the target. First, a hint to the meaning of adding ( $\left.E_{\mathrm{ps}}+E_{\mathrm{exc}}\right)$ to $E_{0}$ may be found in the FBA cross section for the elastic scattering from the Yukawa potential. The Yukawa potential is a screened Coulomb potential,

$$
v(r)=-(Z / r) \exp (-r / \beta)
$$

where $r$ is the radial coordinate, $Z$ is the atomic number and $\beta$ is the "range" of interaction with dimension of length. Using plane waves for the incident electron, the integrated cross section for elastic scattering is

$$
\sigma_{\text {Born }}=16 \pi Z^{2} \beta^{4} /\left[1+4 k^{2} \beta^{2}\right]
$$

where $k$ is the momentum of the incident electron in atomic units. After writing $\beta=b a_{0}$ and noting that $\left(k a_{0}\right)^{2}=T / R$, Eq. (4) becomes

$$
\sigma_{\text {Born }}=4 \pi a_{0}^{2} R Z^{2} b^{2} /\left[T+R / 4 b^{2}\right],
$$

which has the $T$ in the denominator shifted by a Constant with the dimension of energy. This analogy with the Eq. (2), suggests that the constant $\left(E_{\mathrm{ps}}+E_{\mathrm{exc}}\right)$ may be related to the shielding of the nuclear charge by the bound electrons of the target (at higher energies the integral cross sections for elastic and inelastic positron-target scattering are expected to be similar). Is relevant to note that our cross sections using the SBP approach are consistent with sophisticated methods and a particular interest, is to check if the SBP approach is capable, for instance, of describing cross sections consistent with the literature (without Ps channel).

\section{Results and discussion}

We present the calculated integral cross sections (ICS) for $\mathrm{H}, \mathrm{He}, \mathrm{Be}, \mathrm{Mg}$, and $\mathrm{Hg}$ atoms by positron scattering and the cross sections are compared to other theories and experiments (these atoms are chosen because recent experimental data for electron scattering are described in the literature, and hence well suited as a benchmark for our method using positron as incident particle). For all targets we used the theoretical $f$ deduced by Kim [5] and the wave functions for the excited electronic states were all generated with the improved virtual orbital [4].

\section{H-atom}

We performed calculations for the Hydrogen atom as our starting point and the ICS for transition 1s-2s $\left(E_{\text {exc }}=\right.$ $10.2 \mathrm{eV}$, and $E_{\mathrm{ps}}=6.8 \mathrm{eV}$ ) shown in Fig. 1. As observed the FBA cross section for this electronic transition is large, and the SBP cross section is very similar with the multi-state close coupling (MCC) calculation of Walters [6]. We can note also in Fig. 1 that the SBP approach not only reduces the cross section magnitude at low energy, but also shifts the peak to the high energy than the peak of the FBA, while keeping the high energy validity of the FBA intact.

In Fig. 2, the integral cross sections (ICS) using the SBP approach for the 1s-2p excitation of $\mathrm{H}$ atom are compared to 


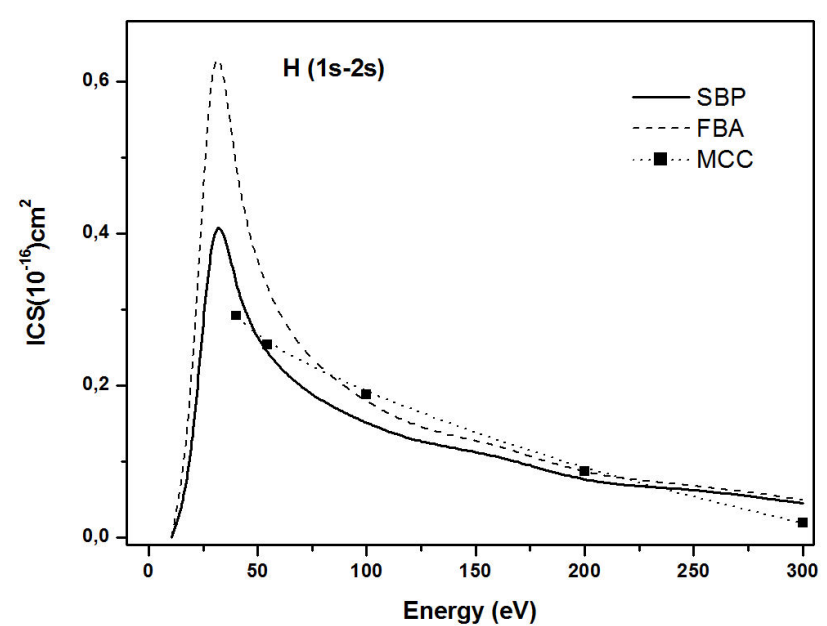

FIGURE 1. Integral cross sections (ICS) for positron impact excitation of the $\mathrm{H}$ atom (1s-2s). Solid line, SBP approach; dashed line, FBA; square with dashed line, MCC [6].

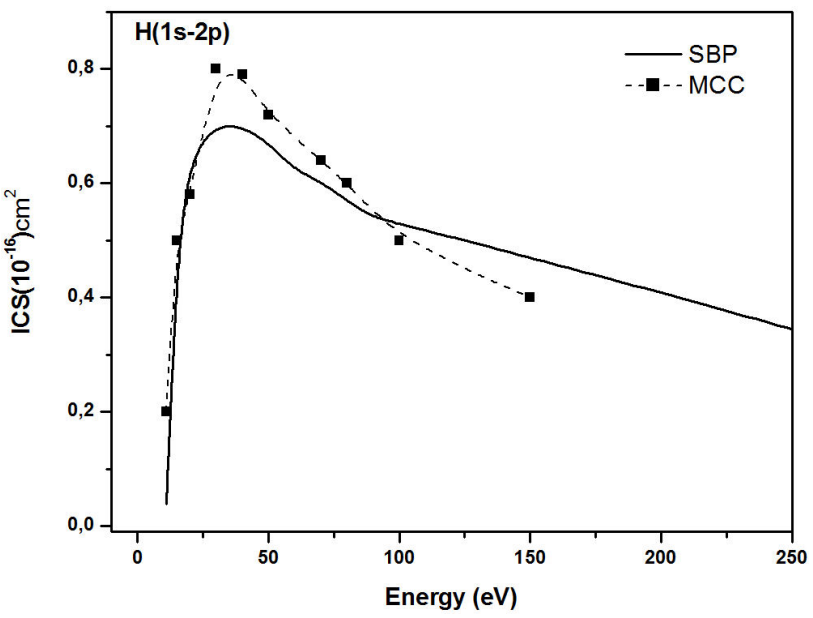

FIGURE 2. Integral cross sections for positron impact excitation of the $\mathrm{H}$ atom (1s-2p). Solid line, SBP; dashed line, FBA; square with dashed line, MCC method [6].

the MCC [6] method. Note in Fig. 2 a good level of agreement between the SBP approach and the MCC method [6].

In the high energy region, the polarization interaction, changing with the impact velocity, becomes relatively weaker. For the static potential, FBA predicts equal cross sections for positron and electrons [3]. This convergence (cross sections) for positron and electrons can be observed using Eq. (2), i.e,

$$
\sigma_{\text {positron }} \approx \sigma_{\text {electron }} \approx \sigma_{\text {Born }}
$$

Figure 3 shows this effects on the electronic excitation process of $\mathrm{H}$ atom. Cross sections for positron scattering using the SBP approach (1s-2p, state) close with cross sections for electron scattering at high energies, without losing its wellknown validity at high $E_{0}$, Eq. (4). As observed, the SBP approach is very similar with BE $f$-scaling [5] and as expected, predicts equal cross sections for positrons and electrons.

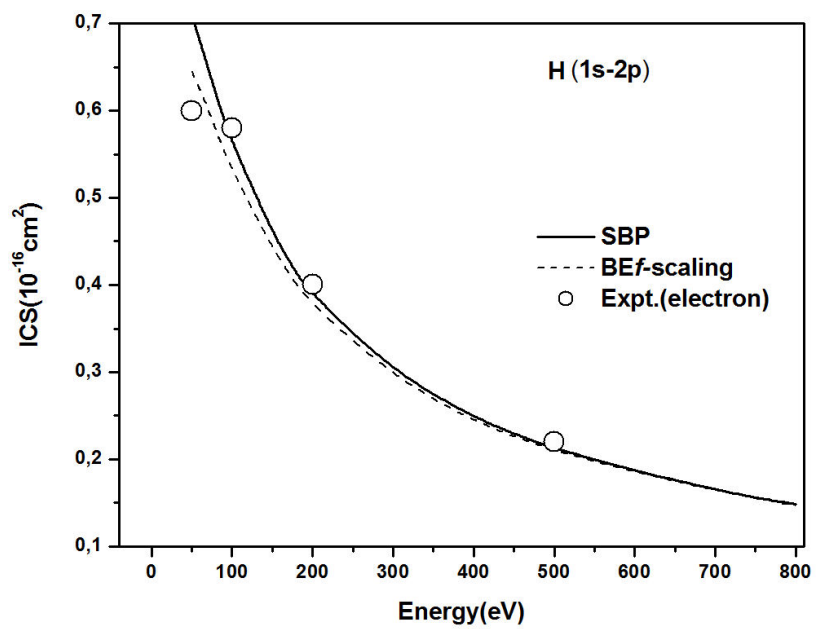

FIGURE 3. Integral cross sections for positron impact excitation of the $\mathrm{H}$ atom (1s-2p). Solid line, SBP approach; dashed line, BE $f$ scaling (electron scattering) [5]; circle, experimental data (electron) [5].

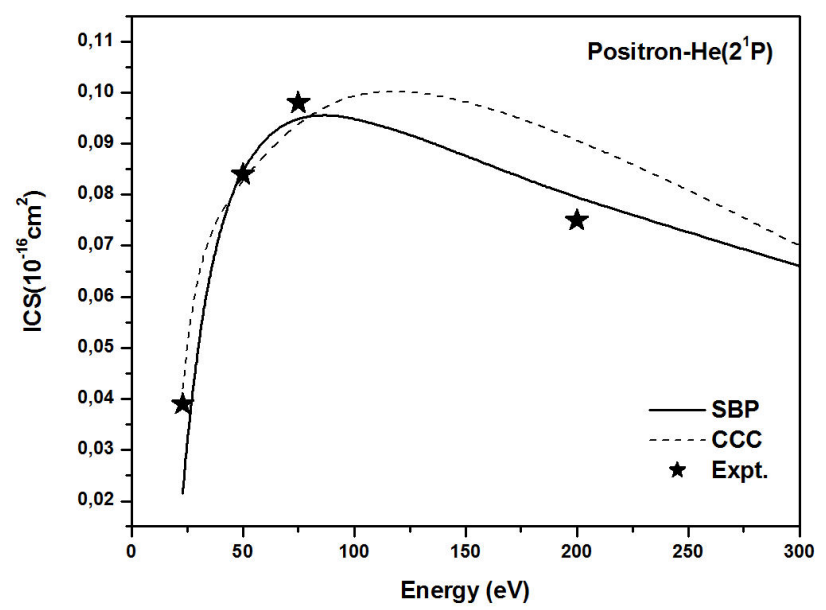

FIGURE 4. Integral cross sections (ICS) for positron impact excitation of the He atom $\left(1 \mathrm{~s}-2 \mathrm{p}^{1} \mathrm{P}\right)$. Solid line, SBP approach; dashed line, CCC method [7]; star, experimental data [8].

\section{He-atom}

Figure 4 shows the integral cross sections (ICS) for $e^{+}-\mathrm{He}$ excitation (1s-2p), $E_{\mathrm{exc}}=21.21 \mathrm{eV}$, and $E_{\mathrm{ps}}=17.78 \mathrm{eV}$. As noted, the SBP approach is reasonably consistent with the convergent close coupling method (CCC) [7] and experimental data [8].

Figure 5 shows the relationship between the states $2^{1} \mathrm{~S}$, and $2^{1} \mathrm{P}$ for $\mathrm{He}$ atom. Integral cross sections for positron excitation of the He atom are compared. As expected the $2{ }^{1} \mathrm{P}$ state is dominant, and hence well suited as a benchmark using the SBP model.

Figure 6 shows a similar agreement between the SBP approach and the sophisticated CCC method [10] for the $2^{1} \mathrm{~S}$ state of $\mathrm{He}$ atom. Again the SBP model is consistent with experimental data [8]. The performance of the SBP approach 


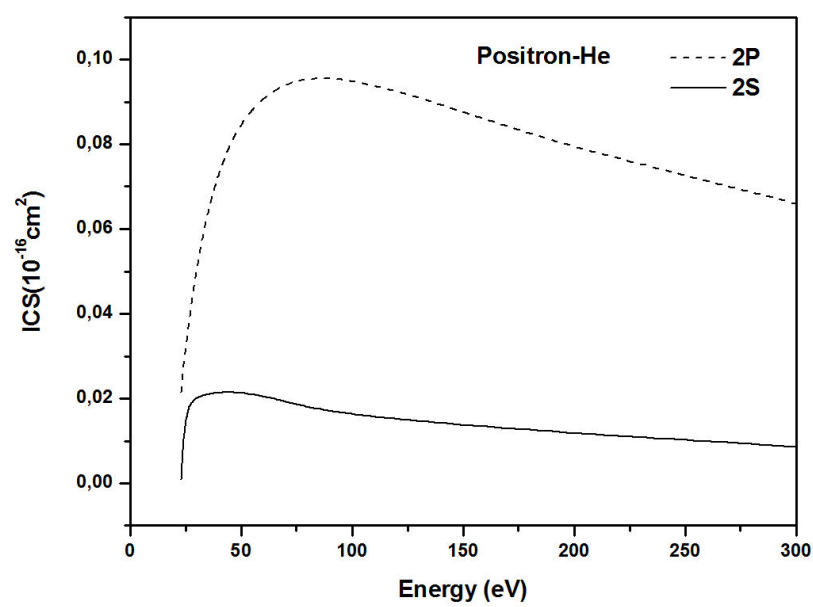

FIGURE 5. Integral cross sections (ICS) for positron impact excitation of the He atom $\left(2^{1} \mathrm{~S}\right.$, and $\left.2 \mathrm{p}^{1} \mathrm{P}\right)$ using SBP approach.

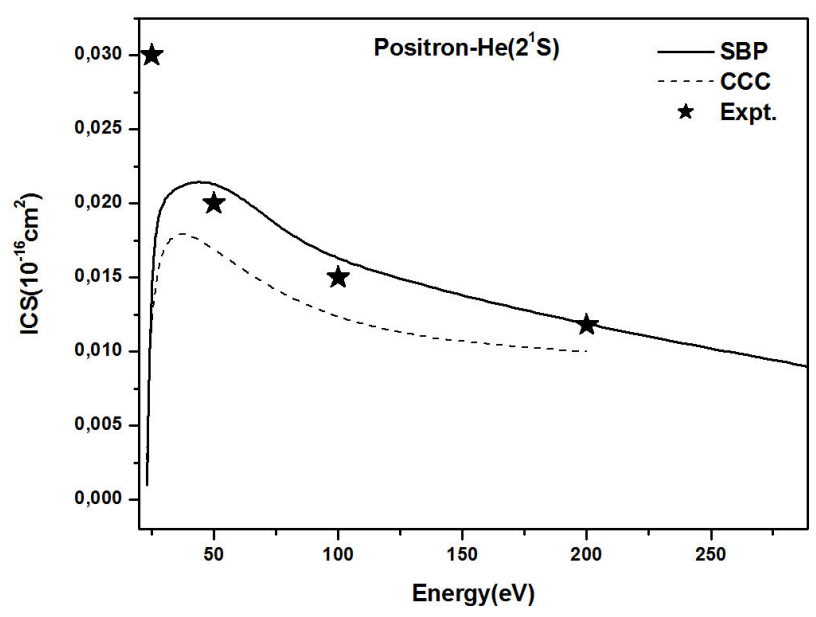

FIGURE 6. Integral cross sections (ICS) for positron impact excitation of the He atom $\left(1 \mathrm{~s}-2^{1} \mathrm{~S}\right)$. Solid line, SBP approach; dashed line, CCC method [7]; star, experimental data [8].

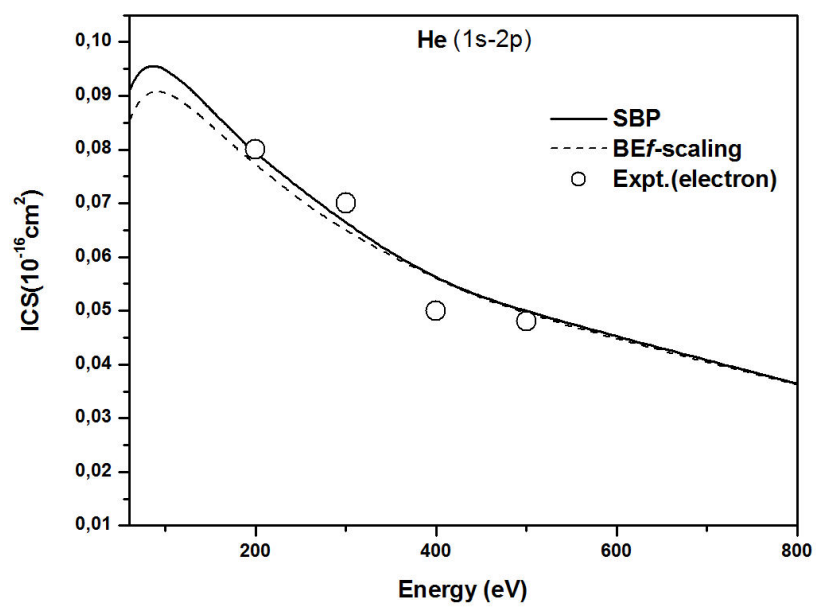

FIGURE 7. Integral cross sections (ICS) for positron impact excitation of the He atom $\left(1 \mathrm{~s}-2^{1} \mathrm{P}\right)$. Solid line, SBP approach; dashed line, $\mathrm{BE} f$-scaling [5]; circle, experimental data for electron scattering [5].

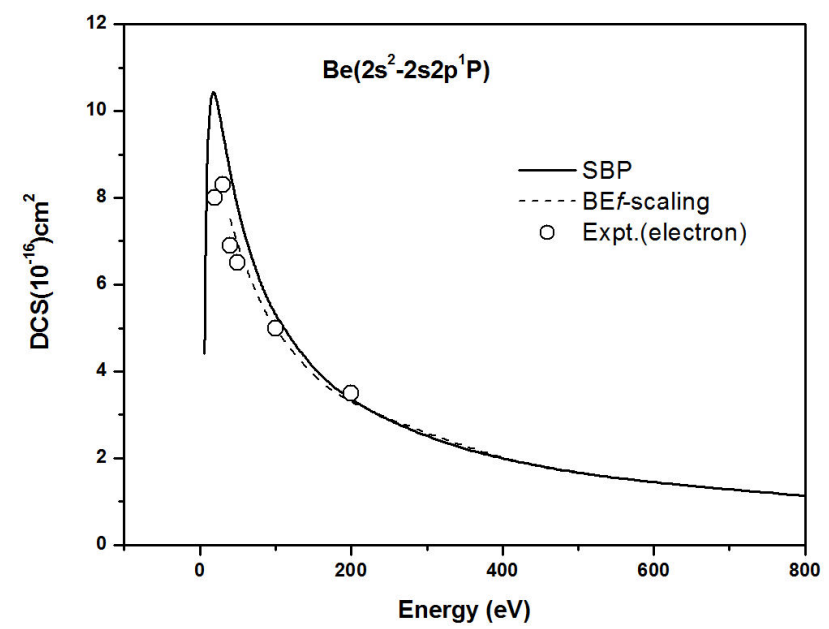

FIGURE 8. Integral cross sections (ICS) for positron impact excitation of the Be atom $\left(2 \mathrm{~s} 2-2 \mathrm{~s} 2 \mathrm{p}^{1} \mathrm{P}\right)$. Solid line, SBP approach; dashed line, $\mathrm{BE} f$-scaling [5]; circle, experimental data for electron scattering [5].

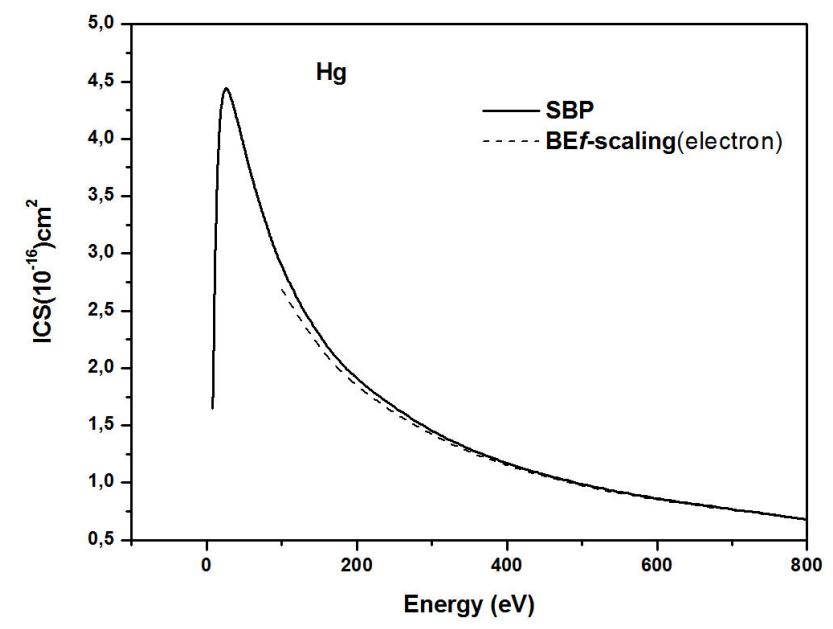

FIGURE 9. Integral cross sections (ICS) for positron impact excitation of the $\mathrm{Hg}$ atom $\left(6 \mathrm{~s} 2-6 \mathrm{~s} 6 \mathrm{p}^{1} \mathrm{P}\right)$. Solid line, SBP approach; dashed line, $\operatorname{BE} f$-scaling [5].

for He atom is remarkable, particularly in view of the simplicity of the scaling.

As in Fig. 3, the Fig. 7 shows the SBP approach compared with $\mathrm{BE} f$-scaling for electron scattering. Note again the good agreement at high energies. As expected, the SBP is applicable at high energies, i.e., the cross sections suggests that the SBP approach does offer a very useful alternative.

\section{Be atom}

Figure 8 presents the SBP approach cross sections for the Be atom $\left(2 \mathrm{~s} 2-2 \mathrm{~s} 2 \mathrm{p}^{1} \mathrm{P}\right.$ sate, $E_{\mathrm{exc}}=5.27 \mathrm{eV}$, and $\left.E_{\mathrm{ps}}=2.52 \mathrm{eV}\right)$. In the absence of experimental data and theoretical studies, our results are compared with $\mathrm{BE} f$-scaling (electron) and experimental data (electron) [5]. As noted the SBP again close with $\operatorname{BE} f$-scaling for electron scattering at high energies. 


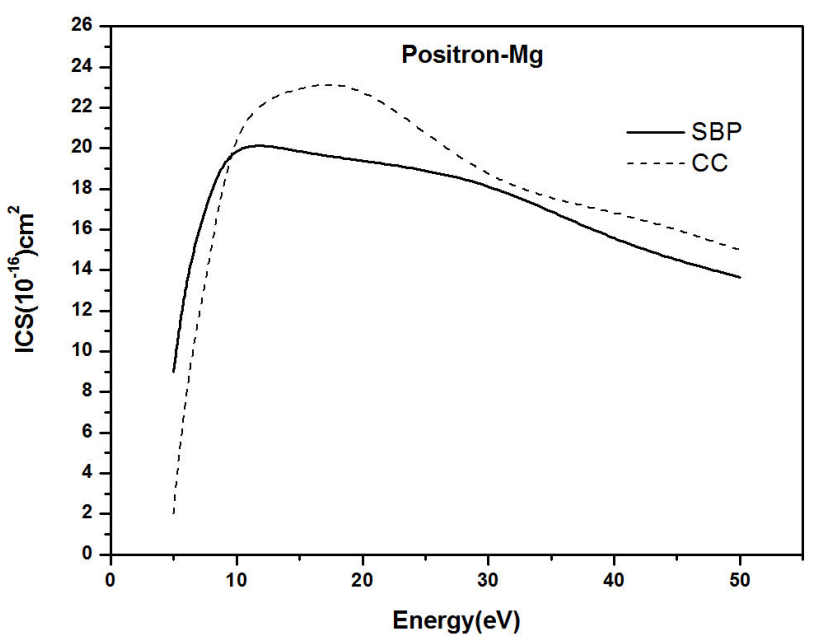

FIGURE 10. Integral cross sections (ICS) for positron impact excitation of the $\mathrm{Mg}$ atom. Solid line, SBP approach; dashed line, theoretical method of Ref. 9.

\section{Hg atom}

Figure 9 shows the SBP approach cross sections for the $\mathrm{Hg}$ atom $\left(6 \mathrm{~s} 2-6 \mathrm{~s} 6 \mathrm{p} 1 \mathrm{P}, E_{\mathrm{exc}}=6.70 \mathrm{eV}\right.$, and $\left.E_{\mathrm{ps}}=3.63 \mathrm{eV}\right)$. In the absence of experimental data and theoretical studies, our results are compared with $\operatorname{BE} f$-scaling (electron) and experimental data (electron). As noted the SBP approach again close with $\mathrm{BE} f$-scaling at high energies.

\section{Mg atom}

The $\mathrm{Mg}$ atom has a ground state Ps formation threshold of only $0.8 \mathrm{eV}$ which is closer to zero energy than any ground or excited state Ps formation thresholds for the alkali atoms. In addition to this, $\mathrm{Mg}$ is a member of the alkaline earth metals family of elements which has never been investigated in an $e^{+}$scattering experiment. As cited before in the SBP approach, the Ps-channel is not considered but the Fig. 10 clearly demonstrates the utility of the present SBP approach, even without Ps-channel the cross sections are consistent with the sophisticated close coupling method (without Pschannel) [9]. As observed again, the SBP approach does offer a very useful alternative method.

\section{Conclusions}

A study of various transitions in positron scattering with ground-state of atoms has been performed using the scaling Born positron (SBP) approach, without the Ps-channel. The inelastic cross sections scattering are reported for low, intermediate and high energies. We observe that the inelastic cross sections using the SBP approach become relatively well converged with sophisticated methods. The SBP approach is a simple representation of the Born cross sections and in this sense, the present approach retains much of the utility of the original Born model and requires a relatively small amount of computing effort. The SBP described here will facilitate the calculation of integrated excitation cross sections for many atoms, making the formulas ideally suited for molecules and other applications where cross sections for a wide range of incident energies are required. The SBP approach is relatively simple compared to state-of-the-art ab initio theories.
1. M. Charlton, J.W. Humberston, Positron Physics (Cambridge University Press, Cambridge, 2001).

2. T.J. Murphy, C.M. Surko, Phys. Rev. A 465696 (1992); C.M. Surko, G.F. Gribakin, S.J.Buckman, J.Phys. (2005) B38 R57.

3. F. Salvat, Phys. Rev. A 68 (2003) 012708.

4. J.L.S.Lino, Rev.Mex.Fis. 63 (2017) 303; J. L. S. Lino, Rev. Mex. Fis. 62 (2016) 596; J.L.S. Lino, Chin. J. Phys. 54 (2016) 223.
5. Y.K. Kim, Phys. Rev. A. 64 (2001) 0327131; Y.K.Kim, Phys. Rev. A 65 (2002) 022705.

6. H.R.J. Walters, Phys. Rep. 116 (1984).

7. C.Y.Jun, Z.Y. Jun, Chin. J. Phys. 19 (2010) 063405.

8. H. Wu , I. Bray, D.V. Fursa, A.T. Stelbovics, J. Phys. B 37 (2004) 1165.

9. See, T.S. Stein et al., Nucl. Instr. Meth.Phys. Res.B 143 (1998) 68. 